\title{
Peroxiredoxin-1 as a prognostic factor in patients with ovarian cancer
}

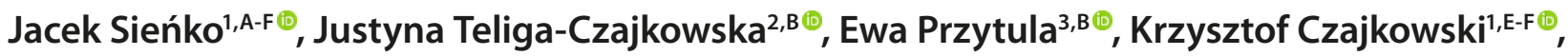 \\ Roman Smolarczyk ${ }^{4, E-F} \oplus$, Dominika Nowis ${ }^{5,6, A-B, D-F} \oplus$
}

1 2nd Department of Obstetrics and Gynecology, Medical University, Warsaw, Poland

${ }^{2}$ Department of Obstetrics and Gynecology Didactics, Medical University, Warsaw, Poland

${ }^{3}$ Department of Pathology, The Medical Center of Postgraduate Education, Warsaw, Poland

${ }^{4}$ Department of Gynecological Endocrinology, Medical University, Warsaw, Poland

${ }^{5}$ Department of Immunology, Cenrtre for Biostructure Research, Medical University, Warsaw, Poland

${ }^{6}$ Laboratory of Experimental Medicine, Centre of New Technologies, Warsaw University, Poland

A - Research concept and design, B - Collection and/or assembly of data, C - Data analysis and interpretation,

$D$ - Writing the article, E - Critical revision of the article, F- Final approval of article

Sieńko J, Teliga-Czajkowska J, Przytula E, Czajkowski K, Smolarczyk R, Nowis D. Peroxiredoxin-1 as a prognostic factor in patients with ovarian cancer. Ann Agric Environ Med. 2019; 26(3): 415-419. doi: 10.26444/aaem/105899

\begin{abstract}
Introduction and objective. Peroxiredoxin-1 (PRDX-1) belongs to a family of antioxidant enzymes and has proved to be a versatile molecule regulating cell growth, differentiation and apoptosis. PRDX1-regulated signaling pathways play an important role in the progression and metastasis of human tumours, especially in breast, esophageal and lung cancers. The aim of the study was to evaluate the expression of PRDX-1 in ovarian cancer tissues, and to test the clinical value of PRDX-1 as a prognostic factor in this malignancy.

Materials and method. PRDX-1 expression was assessed by automated immunohistochemistry in tumours taken from 55 patients with ovarian cancer during primary surgery. Specimen were formalin-fixed and preserved in paraffin-embedded blocks. The results were correlated with clinicopathological data.

Results. A high expression of PRDX-1 was observed in $20 \%$ of cases, and was associated with worse compliance to chemotherapy protocol $(\mathrm{P}<0.002)$, worse response to chemotherapy $(\mathrm{P}<0.04)$, and higher levels of $C A 125$ after the $1^{\text {st }}$ line treatment $(P<0.004)$. PRDX-1 positive subjects had a significantly lower 5 -year disease-free survival $(9.1 \%$ vs. $42.6 \%, P<0.01)$ and a lower 5 -year overall survival (9.1\% vs. 56.7\%; $\mathrm{P}<0.002)$. Multivariate analysis showed that a high expression of PRDX-1 is an independent prognostic factor of poor, overall survival $(\mathrm{P}<0.002)$ and a disease-free survival $(\mathrm{P}<0.01)$.

Conclusion. Results of the study show that PRDX-1 expression in tumour tissues can be another biomarker of prognosis in patients with ovarian cancer.
\end{abstract}

Key words

Peroxiredoxin-1, PRDX-1, ovarian cancer, overall survival, disease free survival

\section{INTRODUCTION}

Ovarian cancer is the fifth leading cause of cancer-related death in females in developed countries [1]. Lack of clear early symptoms or sufficiently sensitive screening tests is the reason that $80 \%$ of patients are diagnosed with ovarian cancer when it is already widespread within the peritoneal cavity [2]. Despite some improvement in treatment, the 5-year survival rate in stages III and IV does not exceed $41 \%$ and $20 \%$, respectively [2]. The standard therapeutic approach for ovarian cancer is upfront surgery followed by a combination of platinum and taxane-based chemotherapy. Outcome is strictly related to the degree of treatment protocol completed [3]. The risk of severe complications often limits optimal surgery $[4,5]$, and drug resistance and toxicity are the most important obstacles for effective chemotherapy $[6,7]$. Those problems could probably be at least partly overcome by the selection of appropriate treatment regimens dependent on prognostic / predictive factors. Very few biomarkers, e.g. BRCA mutation, influence specific treatment selection in

Address for correspondence: Jacek Sieńko, 2nd Department of Obstetrics and Gynecology, Medical University of Warsaw, Karowa 2, 00-315, Warsaw, Poland E-mail: jacek.sienko@wum.edu.pl

Received: 28.02.2019; accepted: 21.03.2019; first published:01.04.2019 ovarian cancer [8]. Thus, the search for novel tumour markers that would play a role as prognostic factors seems to be one of important goals to help customize ovarian cancer treatment which, in turn, could result in better prognosis.

Peroxiredoxin-1 (PRDX-1) is a member of a family of thiol-specific antioxidant proteins that influence hydrogen peroxide levels and mediate signal transduction pathways [9]. PRDX-1 was first reported as an antioxidant enzyme, but its physiological role in oxidization-reduction balance remains unclear because of its high susceptibility to oxidative stress [10]. It has a regulatory function in cell proliferation, differentiation and apoptosis [11]. PRDX-1 oligomers, which function as chaperones under oxidative stress conditions, can interact with the c-Myc oncogene and suppress its transcriptional activity, in turn inhibiting tumourigenesis and promoting tumour cell apoptosis $[12,13,14]$. On the other hand, in certain circumstances, PRDX-1 may act as an oncogene and suppress tumour cell death by directly associating with transcription factors, such as nuclear factor kappa B (NF- $\kappa$ B) and androgen receptor (AR) $[15,16]$. PRDX1 is over-expressed in many human malignant tumours, including lung, breast, urinary, esophageal, hepatocellular and endometrial carcinomas $[17,18,19,20,21,22]$. However, specific role of PRDX-1 in mammary carcinomas is 
controversial. PRDX-1 functions as a chaperone to enhance the transactivation potential of NF- $\kappa \mathrm{B}$ in ER-breast cancer cells, and then suppresses tumour cell death [18]. High expression of PRDX-1 in human breast cancer is associated with higher tumour grade and a higher risk of local recurrence after radiotherapy $[25,26]$. Nevertheless, biomarker studies have demonstrated that PRDX-1 protects estrogen receptors $(\mathrm{ER} \alpha)$ from oxidative stress-induced suppression, and is a protein marker of favourable prognosis in mammary tumours [27]. As in breast cancer, the role of PRDX-1 in esophageal cancer remains ambiguous. It promotes tumourigenesis by functioning as an 'accomplice' of certain oncoproteins or by the activation of its antioxidant enzyme, but it may also play a role as a tumour suppressor through the stimulating of a cyclin-dependent kinase inhibitor - p21 - over-expression [10, 27]. Riddell et al. have shown that the interaction of PRDX1 with the Toll-like receptor 4 (TLR4) stimulates tumour angiogenesis via up-regulation of vascular endothelial growth factor (VEGF) expression in prostatic carcinoma, suggesting that it plays a certain role in malignancy growth, invasion and metastasis [19]. In a previous study, the authors compared the PRDX family over-expression in ovarian cancer; however, the study was performed at the mRNA level [A1]. The aim of the current study is to investigate peroxiredoxin-1 at the protein level as a potential biomarker for prognosis in patients with ovarian cancer.

\section{MATERIALS AND METHOD}

The study was conducted under the approval of the local Ethics Committee and performed in accordance with the World Medical Association Declaration of Helsinki. All subjects provided written informed consent.

The study included 55 patients with ovarian cancer, primary treated in the Princess Anna Mazowiecka Hospital (Medical University of Warsaw) between 2003-2010. Tissue samples were collected for immunohistochemistry from tumour tissues taken during surgical resection, formalin-fixed and preserved in paraffin-embedded blocks. Clinicopathological data were obtained from medical records. The patients were observed 71-4,848 day from the initial treatment (mean $1807+/-1,576$ days). After excluding the patients who died during the observation, the mean follow-up time was $2,936+/$ 1,544 days (213-4848 days).

Immunohistochemical staining for PRDX-1. Sections (4- $\mu \mathrm{m}$ thick) obtained from cancerous tissues were deparaffinized in xylene and rehydrated in descending gradient alcohols. For antigen retrieval, EnVision ${ }^{\mathrm{TM}}$ FLEX Target Retrieval Solution, High pH was used according to the manufacturer's instruction. Immunohistochemical (IHC) reaction was performed in a Dako Autostainer (Agilent Technologies) using an EnVision FLEX Mini Kit. High pH (Agilent Technologies) PRDX1 was detected with rabbit polyclonal anitbody (Cat No HPA007730, Sigma-Aldrich). IHC staining was assessed by an experienced pathologist. The samples were categorized as negative if PRDX-1 expression was either imperceptible or very weak. A positive result was only assigned to a strong expression of PRDX-1.

Statistical analysis. The results were presented as mean \pm standard deviation (SD). Significant statistical differences between groups were assessed applying the chi-square test, exact Fisher test or Student's $t$ test. The KaplanMeier method was employed to plot survival curves, and differences in survival were compared using the log-rank test. The Cox regression model was used to ascertain the value of independent prognosis for postoperative patients with ovarian cancer. $\mathrm{P}<0.05$ was considered statistically significant.

\section{RESULTS}

A strong expression of PRDX-1 was found in 11 (20\%) cases of ovarian cancer which enabled them to be classified in the PRDX-1 positive group. In the PRDX-1 negative group $(\mathrm{N}=44), 31$ cases presented a very weak/minimal expression of PRDX-1. PRDX-1 expression was present in the cytoplasm of cancerous cells, but not in the stroma. The clinicopathological characteristic of the groups according to PRDX-1 expression is presented in Table 1.

Table 1. Clinicopathological characteristic of the PRDX-1 negative and positive groups

\begin{tabular}{lccc}
\hline Age [years] & $54.4+/-9.78$ & $59.7+/-10.9$ & 0.12 \\
\hline - Histological type n (\%) & $20(45.5 \%)$ & $7(63.6 \%)$ & \\
- Serous & $14(31.8 \%)$ & $2(18.2 \%)$ & 0.68 \\
- Endometrioid & $7(15.9 \%)$ & $1(9.1 \%)$ & \\
- Clear cell Mucinous & $3(6.8 \%)$ & $1(9.1 \%)$ & \\
\hline Stage n (\%) & $21(50 \%)$ & $2(18.2 \%)$ & 0.07 \\
I & $3(6.8 \%)$ & $0(0 \%)$ & \\
II & $19(43.2 \%)$ & $9(81.8 \%)$ & \\
III & $0(0 \%)$ & $0(0 \%)$ & \\
IV & & & \\
\hline Grade $n$ (\%) & $6(15.8 \%)$ & $2(25 \%)$ & 0.52 \\
1 & $20(52.6 \%)$ & $5(62.5 \%)$ & \\
2 & $12(31.6 \%)$ & $1(12.5 \%)$ & \\
3 & $851.9+/-1192.4$ & $1048.6 .4+/-1375.3$ & 0.64 \\
\hline CA 125 before initial treatment & $15.5+/-6.5$ & $13.1+/-13.8$ & 0.40 \\
\hline U/L] & & & \\
\hline Largest tumour size [cm] & & & \\
\hline
\end{tabular}

Values are mean+/-SD or present a number (\%) of cases in groups. Student's $t$ test or exact Fisher test were applied, respectively

The groups did not differ in terms of well-known prognostic factors, such as age, tumour stage and grade, CA 125 concentration before treatment or the largest tumour size. The patients received standard treatment that included surgery and adjuvant platinum-based chemotherapy. The results of the first-line treatment are presented in Table 2.

Optimal results of surgery defined as no visible residual volume was achieved in $47.7 \%$ of PRDX-1 negative and $18.2 \%$ of PRDX-1 positive patients (NS). 44 (100\%) PRDX-1 negative but only 8 PRDX-1 positive patients $(72.7 \%)$ received at least 6 courses of platinum derivatives (Cisplatin or Carboplatin) $(\mathrm{P}<0.002)$. The differences in response to the first line treatment were significant if stratified as any response vs. progression $(\mathrm{P}<0.04)$. CA 125 level assessed after the end of the first line chemotherapy treatment was significantly lower in the PRDX-1 negative group (5.73+/-3.77 vs. 78.25+/124.62 U/L, $\mathrm{P}<0.004)$.

Survival analyses. Kaplan-Meier curves (Fig. 1) analysis was used to compare the overall survival (OS) and disease- 
Table 2. Correlation between PRDX-1 expression and the clinical results achieved during treatment

\begin{tabular}{|c|c|c|c|}
\hline & $\begin{array}{c}\text { PRDX-1 } \\
\text { negative } \\
\mathrm{N}=44\end{array}$ & $\begin{array}{l}\text { PRDX-1 } \\
\text { positive } \\
\mathrm{N}=11\end{array}$ & $P$ \\
\hline Optimal surgery (no residual volume) & $21(47.7 \%)$ & $3(18.2 \%)$ & 0.081 \\
\hline $\begin{array}{l}\text { At least } 6 \text { cycles of chemotherapy } \\
\text { completed }\end{array}$ & $44(100 \%)$ & $8(72.7 \%)$ & 0.002 \\
\hline Response to treatment & & & 0.052 \\
\hline - Complete remission & $33(75 \%)$ & $4(36.4 \%)$ & 0.04 \\
\hline - Partial remission & $6(13.6 \%)$ & $3(27.2 \%)$ & \\
\hline - Stabilization & $1(2.3 \%)$ & $0(0 \%)$ & \\
\hline - Progression & $4(9.1 \%)$ & $4(36.4 \%)$ & \\
\hline \multirow[t]{2}{*}{ Any response vs. progression } & $40(90.9 \%)$ & $7(63.6 \%)$ & \\
\hline & $4(9.1 \%)$ & $4(33.4 \%)$ & \\
\hline CA 125 after first-line treatment [U/L] & $5.73+/-3,77$ & $78.25+/-124.62$ & 0.004 \\
\hline
\end{tabular}

Values are mean+/-SD or present a number (\%) of cases in groups. Student's $t$ test or exact Fisher test were applied, respectively

free survival (DFS) between PRDX-1 positive and negative patients. PRDX-1 positive subjects had a significantly higher risk of recurrence (DFS $9.1 \%$ vs. $42.6 \%$ within 5 years; $\mathrm{P}<0.01$ ) and a lower probability of a 5 -year survival $(9.1 \%$ vs. $56.7 \%$; $\mathrm{P}<0.002$ ), compared to PRDX-1 negative patients. Univariate analysis showed that PRDX-1 expression, tumour stage (early vs. advanced ovarian cancer), histological type (serous vs. nonserous) and CA 125 before treatment (twice elevated above reference value) have prognostic value on OS and DFS (Tab. 3).

Multivariate analysis revealed that PRDX-1 is an independent marker of poor prognosis, both for DFS and OS (Tab. 4). Tumour stage was also an independent predictor of OS and DFS and CA 125 elevated at least twice above reference value was the third independent prognostic factor for DFS, but not for OS.

\section{DISCUSSION}

PRDX-1 was found to be over-expressed in many different cancers $[18,19,29]$. A high expression of PRDX-1 was usually associated with poor outcomes $[10,24,24,26]$. In this study, 1 in 5 cancers presented a high expression of PRDX-1. In the study by Cai et al., $74.4 \%$ of cancerous tissues of pancreatic cancer tested positive for PRDX-1 [28]. This would be comparable with the presented results (76.4\%) if the groups
Table 3. Univariate analysis of factors associated with OS and DFS. The Cox regression model

\begin{tabular}{|c|c|c|c|c|c|c|}
\hline \multirow{2}{*}{ Variable } & \multicolumn{3}{|c|}{ os } & \multicolumn{3}{|c|}{ DFS } \\
\hline & $\mathrm{RR}$ & $95 \% \mathrm{Cl}$ & $\mathrm{P}$ & $\mathrm{RR}$ & $95 \% \mathrm{Cl}$ & $P$ \\
\hline $\begin{array}{l}\text { PRDX-1 negative } \\
\text { vs. positive }\end{array}$ & 0.25 & $0.11-0.55$ & 0.0006 & 0.31 & $0.15-0.67$ & 0.002 \\
\hline $\begin{array}{l}\text { Age }<60 \text { years vs. } \\
>60 \text { years }\end{array}$ & 0.86 & $0.40-1,86$ & 0.71 & 0.22 & $0.38-1.55$ & 0.46 \\
\hline $\begin{array}{l}\text { Tumour size } \\
<20 \mathrm{~cm} \text { vs. }>20 \mathrm{~cm}\end{array}$ & 0.60 & $0.27-1.29$ & 0.19 & 0.65 & $0.32-1.31$ & 0.23 \\
\hline FIGO I-II vs. III-IV & 0.15 & $0.06-0.37$ & 0.00003 & 0.16 & $0.07-0.34$ & 0.000002 \\
\hline Grade $2-3$ vs. 1 & 1.19 & $0.40-3.51$ & 0.75 & 0.84 & $0.32-2.2$ & 0.73 \\
\hline $\begin{array}{l}\text { Non-serous vs. } \\
\text { serous tumour }\end{array}$ & 0.40 & $0.18-0.85$ & 0.02 & 0.33 & $0.17-0.67$ & 0.002 \\
\hline $\begin{array}{l}\text { CA } 125 \text { before } \\
\text { treatment }<70 U / L \\
\text { vs. }>70 U / L\end{array}$ & 0.18 & $0.04-0.77$ & 0.02 & 0.13 & $0.03-0.55$ & 0.005 \\
\hline
\end{tabular}

vs. $>70 \mathrm{U} / \mathrm{L}$

$\mathrm{RR}$ - relative risk of death or release/progression, respectively

Table 4. Multivariate Cox regression analysis of factors associated with OS and DFS

\begin{tabular}{lcccccc}
\hline \multirow{2}{*}{ Variable } & \multicolumn{3}{c}{ OS } & \multicolumn{3}{c}{ DFS } \\
\cline { 2 - 7 } & $\mathrm{RR}$ & $95 \% \mathrm{Cl}$ & $\mathrm{P}$ & $\mathrm{RR}$ & $95 \% \mathrm{Cl}$ & $\mathrm{P}$ \\
\hline PRDX-1 negative vs. positive & 0.28 & $0.11-0.71$ & 0.007 & 0.38 & $0.16-0.90$ & 0.03 \\
\hline FIGO 1-2 vs. 3-4 & 0.24 & $0.08-0.74$ & 0.001 & 0.29 & $0.11-0.75$ & 0.01 \\
\hline $\begin{array}{l}\text { Non-serous vs. serous } \\
\text { tumour }\end{array}$ & 0.81 & $0.36-1.83$ & 0.61 & 0.55 & $0.25-1.21$ & 0.14 \\
\hline $\begin{array}{l}\text { CA 125 before treatment } \\
<70 U / L \text { vs. }>70 U / L\end{array}$ & 0.34 & $0.07-1.62$ & 0.17 & 0.21 & $0.05-0.97$ & 0.04 \\
\hline
\end{tabular}

$\mathrm{RR}$ - relative risk of death or relapse/progression, respectively

were to be stratified in an analogous format, e.g. no vs. any expression of PRDX-1. Statistical analysis of the data obtained in the current study revealed that division of the group into high vs. no/minimal expression of PRDX-1 subgroup, better correlated with outcomes, rather than the inclusion of cases with minimal expression of PRDX-1 to the PRDX-1 positive group. This may be because a strong expression of PRDX-1 reflects a biologically-meaningful over-expression of this protein in the samples.

In this study, a high expression of PRDX-1 was found to be accompanied with worse results in the first line treatment. Although the differences in the percentage of optimal surgery

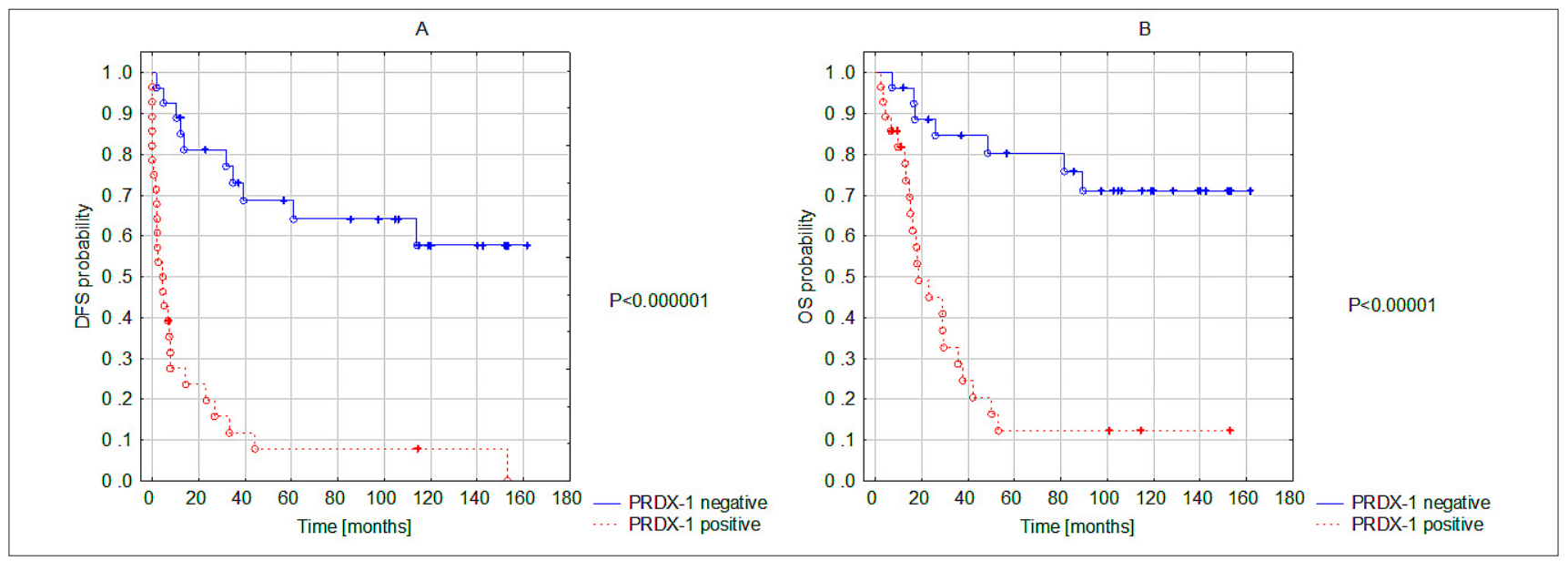

Figure 1. Kaplan-Meier analysis of A. - disease-free survival (DFS) and B. - overall survival (OS). Log rank test. 
were not statistically significant, both its compliance with chemotherapy protocol and response to chemotherapy were poorer. As the main reason for premature chemotherapy termination was progression, it is suspected that high expression of PRDX-1 could be one of the factors involved in the development of resistance to platin. This hypothesis may be supported by the in vitro study by Kalinina et al, which found that cisplatin resistance formation is accompanied by a significant increase in the expression of PRDX1, PRDX2, PRDX3, and PRDX6 genes in human ovarian carcinoma SKOV-3 cells [29]. Kubota et al. identified another member of the PRDX family - peroxiredoxin 2, as a predictive biomarker of response to induction chemotherapy in osteosarcoma [30].

Very little is known about the biological meaning of peroxiredoxins, especially that of PRDX-1, as a prognostic factor in ovarian cancer. Li et al. studied the prognostic values of the peroxiredoxins family in ovarian cancer [31]. The study investigated protein expression profiles in normal ovarian tissues and cancerous ovarian tissues using the Human Protein Atlas database, and made a compilation of several available databases (Gene Expression Omnibus, the Cancer Biomedical Informatics Grid, and The Cancer Genome Atlas) to compare PRDX's expression at the mRNA level. The study found that the elevated expression of PRDX-3, PRDX-5, and PRDX-6 mRNAs showed poorer OS, and the expression PRDX-5 and PRDX-6 also were able to predict poor progression-free survival (PFS). The prognostic value of PRDX-1 in that study was unclear, as the results were either insignificant for all patients or inconsistent for subjects with clinical stage 1 and 2 (better OS and poorer PFS, excluding histological grade 1 for PFS).

This study has demonstrated that high expression of PRDX1 in cancerous tissue is strongly associated with a shorter DFS and OS. These observations are consistent with the results of other studies, excluding those of ovarian cancer tumours $[21,28]$. Sun et al. found that PRDX-1 expression in hepatocellular carcinoma (HCC) cells was significantly associated with numerous parameters of aggressive disease, including an increased tumour size, multiple tumour nodules, microvascular invasion, an advanced Edmondson grade, an incomplete tumour capsule, a higher serum AFP, and advanced stages of the TNM staging system which, in turn, resulted in shorter OS and DFS [21].

In the study by Cai et al., PRDX-1 expression in pancreatic cancer correlated with histological grade, perineural invasion, lymph node metastases, CA 19-9 level and the TNM stage. Moreover, PRDX-1 was a negative and independent predictor of survival and recurrence [28].

There is growing evidence that PRDX-1 is a multidirectionally acting protein involved in tumourigenesis. Not only is it closely related to tumour angiogenesis in pancreatic cancer [29], but it also suppresses proteasome inhibitormediated cell death affecting signal-regulating kinase 1 (ASK1) activation in human thyroid cancer [32]. PRDX-1 equally promotes tumourigenesis by regulating the activity of the mTOR/p70S6K pathway in oesophageal squamous cell carcinoma [34].

As demonstrated, PRDX-1 is an independent prognostic factor in patients with ovarian cancer who have undergone surgery. It makes PRDX-1 a candidate for more comprehensive studies in order to determine its significance as a biomarker useful in planning the strategy of treatment. No study was found that allowed comparison of the presented results of
PRDX-1 staining with another group of ovarian cancer patients. However, the existence of a study suggesting that positive PRDX-1 expression can be an independent predictor of favourable prognosis in breast cancer positive for estrogen receptors, is significant [25].

Total peroxiredoxin expression, including PRDX-1, was also associated with prolonged survival in patients with follicular lymphoma [34]. Ding et al. suggested that both redox and chaperone activity determine the role of PRDX1 in the promotion or suppression of oncogenesis in certain types of cancer [10]. These findings seem to demonstrate the complexity of PRDX-1 functions in different tissues and cancers and force the authors of the current study to limit their predictions on the possible role of this biomarker in other malignancies or experimental models. The main limitation of this study is its relatively small sample size. Therefore, final conclusions should be made with caution until further larger studies are conducted.

\section{CONCLUSIONS}

The study shows that a high expression of PRDX-1 is associated with shorter overall and disease-free survival, and serves as an independent prognostic factor for poor OS and DFS in patients with ovarian cancer.

\section{Acknowledgements}

The work was supported by the Ministry of Science and Higher Education grant (IP2012048172; DN)

\section{REFERENCES}

1. Siegel RL, Miller KD, Jemal A. Cancer statistics. CA Cancer J Clin. 2018; 68(1): 7-30.

2. Torre LA, Trabert B, DeSantis CE, Miller KD, Samimi G, Runowicz CD, et al. Ovarian cancer statistics. CA Cancer J Clin. 2018; 68: 284-296.

3. Elattar A, Bryant A, Winter-Roach BA, Hatem M, Naik R. Optimal primary surgical treatment for advanced epithelial ovarian cancer. Cochrane Database Syst Rev. 2011; 10 (8): CD007565.

4. Kebapci E, Gülseren V, Tugmen C, Gökçü M, Solmaz U, Sert J, et al. Outcomes of patients with advanced stage ovarian cancer with intestinal metastasis. Ginekol Pol. 2017; 88(10): 537-542.

5. Oseledchyk A, Hunold LE, Mallmann MR, Domröse CM, Abramian A, Debald M, et al. Impact of Extended Primary Surgery on Suboptimally Operable Patients with Advanced Ovarian Cancer. Int J Gynecol Cancer. 2016; 26(5): 873-83.

6. Pinato DJ, Graham J, Gabra H, Sharma R. Evolving concepts in the management of drug resistant ovarian cancer: dose dense chemotherapy and the reversal of clinical platinum resistance. Cancer Treat Rev. 2013; 39(2): 153-60.

7. Koo YJ, Lim KT. Toxicity of intraperitoneal chemotherapy and risk factors for severe toxicity in optimally debulked ovarian cancer patients. Taiwan J Obstet Gynecol. 2015; 54(3): 275-9.

8. Ledermann J, Harter P, Gourley C, Friedlander M, Vergote I, Rustin G, et al. Olaparib maintenance therapy in patients with platinum-sensitive relapsed serous ovarian cancer: a preplanned retrospective analysis of outcomes by BRCA status in a randomised phase 2 trial. Lancet Oncol. 2014; 15(8): 852-61.

9. Wood ZA, Schroder E, Robin HJ, Poole LB. Structure, mechanism and regulation of peroxiredoxins. Trends Biochem Sci. 2013; 28 (1): 32-40.

10. Ding C, Fan X, Wu G. Peroxiredoxin 1 - an antioxidant enzyme in cancer. J Cell Mol Med. 2017; 21(1): 193-202.

11. Kim JH, Lee JM, Lee HN, Kim EK, Ha B, Ahn SM, et al. RNA-binding properties and RNA chaperone activity of human peroxiredoxin 1 . Biochem Biophys Res Commun. 2012; 425 (4): 730-734.

12. Rhee SG, Woo HA. Multiple functions of peroxiredoxins: peroxidases, sensors and regulators of the intracellular messenger $\mathrm{H} 2 \mathrm{O} 2$, and protein chaperones". Antioxid Redox Signal. 2011; 15: 781-94. 
13. Hopkins BL, Neumann CA. Redoxins as gatekeepers of the transcriptional oxidative stress response. Redox Biol. 2019; 21: 101104

14. Graves JA, Metukuri M, Scott D, et al. Regulation of reactive oxygen species homeostasis by peroxiredoxins and c-Myc. J Biol Chem. 2009; 284(10): 6520-9.

15. Zhang M, Hou M, Ge L, Miao C, Zhang J, Jing X, Shi N, Chen T, Tang $\mathrm{X}$. Induction of peroxiredoxin 1 by hypoxia regulates heme oxygenase-1 via NF- $\kappa$ B in oral cancer. PLoS One. 2014; 9(8): e105994.

16. Chhipa RR, Lee KS, Onate S, et al. Prxl enhances androgen receptor function in prostate cancer cells by increasing receptor affinity to dihydrotestosterone. Mol Cancer Res. 2009; 7: 1543-52.

17. Rostila A, Puustinen A, Toljamo T, Vuopala K, Lindström I, Nyman TA, et al. Peroxiredoxins and tropomyosins as plasma biomarkers for lung cancer and asbestos exposure. Lung Cancer. 2012; 77(2): 450-9.

18. Cha MK, Suh KH, Kim IH. Overexpression of peroxiredoxin I and thioredoxin 1 in human breast carcinoma. J Exp Clin Cancer Res. 2009; 28: 93

19. Riddell JR, Bshara W, Moser MT, Spernyak JA, Foster BA, Gollnick SO. Peroxiredoxin 1 controls prostate cancer growth through toll-like receptor 4-dependent regulation of tumour vasculature. Cancer Res. 2011; 71(5): 1637-1646.

20. Ren P, Ye H, Dai L, Liu M, Liu X, Chai Y, et al. Peroxiredoxin 1 is a tumour-associated antigen in esophageal squamous cell carcinoma. Oncol Rep. 2013; 30: 2297-303.

21. Sun QK, Zhu JY, Wang W, Lv Y, Zhou HC, Yu JH, et al. Diagnostic and prognostic significance of peroxiredoxin 1 expression in human hepatocellular carcinoma. Med Oncol. 2014; 31(1): 786.

22. Byun JM, Kim SS, Kim KT, Kang MS, Jeong DH, Lee DS, et al. Overexpression of peroxiredoxin- 3 and -5 is a potential biomarker for prognosis in endometrial cancer. Oncol Lett. 2018; 15(4): 5111-5118.

23. Wang X, He S, Sun JM, et al. Selective association of peroxiredoxin 1 with genomic DNA and COX?2 upstream promoter elements in estrogen receptor negative breast cancer cells. Mol Biol Cell. 2010; 21: 2987-95.

24. Woolston CM, Storr SJ, Ellis IO, Morgan DA, Martin SG. Expression of thioredoxin system and related peroxiredoxin proteins is associated with clinical outcome in radiotherapy treated early stage breast cancer". Radiother Oncol. 2011; 100: 308-13.

25. O'Leary PC, Terrile M, Bajor M, Gaj P, Hennessy BT, Mills GB, et al. Peroxiredoxin 1 protects estrogen receptor from oxidative stress induced suppression and is a protein biomarker of favorable prognosis in breast cancer. Breast Cancer Res. 2014; 16: R79.

26. Hoshino I, Matsubara H, Hanari N, Mori M, Nishimori T, Yoneyama Y, et al. Histone deacetylase inhibitor FK228 activates tumour suppressor Prdx1 with apoptosis induction in esophageal cancer cells. Clin Cancer Res. 2005; 11: 7945-52.

27. Sienko J, Gaj P, Czajkowski K, Nowis D. Peroxiredoxin-5 is a negative survival predictor in ovarian cancer. Gin Pol. 2019; 90(1): 1-6.

28. Cai CY, Zhai LL, Wu Y, Tang ZG. Expression and clinical value of peroxiredoxin-1 in patients with pancreatic cancer. Eur J Surg Oncol. 2015; 41(2): 228-35.

29. Kalinina EV, Berezov TT, Shtil' AA, Chernov NN, Glazunova VA, Novichkova MD, et al. Expression of peroxiredoxin 1, 2, 3, and 6 genes in cancer cells during drug resistance formation. Bull Exp Biol Med. 2012; 153(6): 878-81.

30. Kubota D, Mukaihara K, Yoshida A, Tsuda H, Kawai A, Kondo T. Proteomics study of open biopsy samples identifies peroxiredoxin 2 as a predictive biomarker of response to induction chemotherapy in osteosarcoma”. J Proteomics. 2013; 8(91): 393-404.

31. Li S, Hu X, Ye M, Zhu X. The prognostic values of the peroxiredoxins family in ovarian cancer. Biosci Rep. 2018; 38(5).

32. Du ZX, Yan Y, Zhang HY, et al. Suppression of MG132-mediated cell death by peroxiredoxin 1 through influence on ASK1 activation in human thyroid cancer cells. Endocr Relat Cancer. 2010; 17: 553-60.

33. Gong F, Hou G, Liu H, et al. Peroxiredoxin 1 promotes tumourigenesis through regulating the activity of mTOR/p70S6K pathway in esophageal squamous cell carcinoma. Med Oncol. 2015; 32: 455.

34. Peroja P, Haapasaari KM, Mannisto S, Miinalainen I, Koivunen P, Leppä $S$, et al. Total peroxiredoxin expression is associated with survival in patients with follicular lymphoma. Virchows Arch. 2016; 468(5): $623-30$

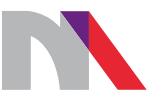

Ministry of Science and Higher Education

Republic of Poland

Generation of the DOI (Digital Object Identifier) - task financed under the agreement No. 618/P-DUN/2019 by the Minister of Science and Higher Education 